\title{
Improvement of coupling suppression by using multi- stacked mushroom structure
}

\author{
Yuki Kawakami $^{1 \mathrm{a})}$, Ryuji Kuse ${ }^{2}$, Toshikazu Hori ${ }^{2}$, \\ and Mitoshi Fujimoto ${ }^{2}$ \\ ${ }^{1}$ National Institute of Technology, Fukui College, \\ Sabae-shi, Fukui 916-8507, Japan \\ ${ }^{2}$ Graduate School of Engineering, University of Fukui, \\ Fukui-shi, Fukui 910-8507, Japan \\ a)y.kawakami@m.ieice.org
}

\begin{abstract}
This paper proposes a multi-stacked mushroom structure to suppress the mutual coupling between monopole antennas, whose height is taller than that of a conventional mushroom structure. Based on moment method analysis results, it is clarified that the multi-stacked mushroom structure can suppress the mutual coupling more than a conventional $2 \mathrm{D}$ arranged structure. Furthermore, the validity of the results is clarified by experiments.
\end{abstract}

Keywords: multi-stacked mushroom structure, monopole antenna, mutual coupling

Classification: Antennas and Propagation

\section{References}

[1] F. Yang and Y. Rahmat-Samii, Electromagnetic Band Gap Structures in Antenna Engineering, Cambridge University Press, Cambridge, 2008. DOI:10.1017/ CBO9780511754531

[2] F. Yang and Y. Rahmat-Samii, "Mutual coupling reduction of microstrip antennas using electromagnetic band-gap structure," IEEE Antennas and Propagation Society International Symposium, vol. 2, pp. 478-481, July 2001. DOI:10.1109/APS.2001.959765

[3] Y. Kawakami, T. Hori, M. Fujimoto, R. Yamaguchi, and K. Cho, "Radiation properties of rectangular microstrip antenna located by EBG structure," Proc. ISAP2007, Niigata, Japan, Aug. 2007.

[4] D. Sievenpiper, L. Zhang, R. F. J. Broas, N. G. Alexopolous, and E. Yablonovitch, "High-impedance electromagnetic surfaces with a forbidden frequency band," IEEE Trans. Microw. Theory Techn., vol. 47, no. 11, pp. 2059-2074, Nov. 1999. DOI:10.1109/22.798001

\section{Introduction}

EBG (Electromagnetic Band-Gap) Structures, which is composed of metal elements arranged at small intervals, have the band gap characteristics. Thus, it can 
reduce undesired electromagnetic waves [1]. From the unique characteristics, EBG structures have been studied to apply to antennas for the suppression of the mutual coupling and the improvement of the radiation property $[2,3]$.

Mushroom structure is the most familiar EBG structure [4]. It is composed of a ground plate, metal patches and short pins. The mutual coupling suppression property by a conventional, two-dimensionally arranged, mushroom structure have been already clarified. However, when a height of antenna is taller than that of the mushroom structure, the coupling will be little suppressed because electromagnetic waves propagate above the mushroom structure.

In this paper, a multi-stacked mushroom structure is proposed to suppress the mutual coupling between monopole antennas whose height is taller than that of conventional mushroom structure. It is shown by moment method analysis that the proposed structure can suppress the coupling more than conventional mushroom structures. Furthermore, the validity of the results is clarified by measurement.

\section{Proposed multi-stacked mushroom structure}

Fig. 1 shows an analysis model of proposed mushroom structure. Fig. 1 shows the analysis model of monopole antennas with the proposed mushroom structure.

As shown in Fig. 1, mushroom structures are located between monopole antennas. The distance $d$ between monopole antennas is equal to $\lambda_{0}$. Here, the wavelength $\lambda_{0}$ is that of the wave at frequency $f_{0}$. The frequency $f_{0}$ is the resonant frequency of the monopole antennas. The number of mushroom elements arranged in $x, y$ and $z$ axis are defined as the number of rows $N_{R}$, columns $N_{C}$ and stacks $N_{S}$, respectively. In this paper, the number of rows $N_{R}$, columns $N_{C}$ and stacks $N_{S}$ is 3, 6 and 3. The mushroom elements in second or third stack are shorted on a same metal plate. Only the elements in second stack are located inversely. The distance $d_{m u s h}$ is the distance of mushroom elements between second and third stack.

The parameters of mushroom structure $T, w$, and $h$ are the period of arrangement, the width of a metal patch and the distance between the patch and the ground

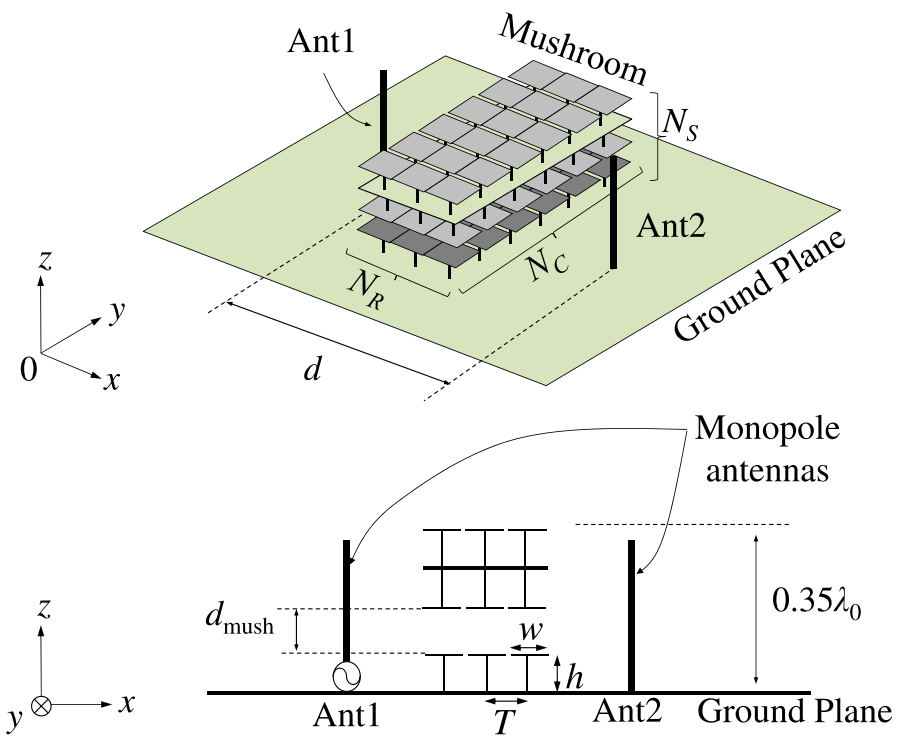

Fig. 1. Analysis model of proposed mushroom structure. 
plane, respectively. In this paper, the parameters of $T, w$, and $h$ are fixed $0.21 \lambda_{0}$, $0.20 \lambda_{0}$ and $0.07 \lambda_{0}$. This mushroom structure has the band gap characteristics from $0.6 f_{0}$ to $1.2 f_{0}$.

In the following section, the mutual coupling suppression property by proposed mushroom structures is calculated by using the moment method.

\section{Mutual coupling suppression property by multi-stacked mush- room structure}

Fig. 2 shows the mutual coupling suppression property of a multi-stacked mushroom structure. Fig. 2(a) shows the frequency response of the normalized mutual coupling $C_{21} / C_{21 \text { ref }}$, and Fig. 2(b) shows the relation between the normalized

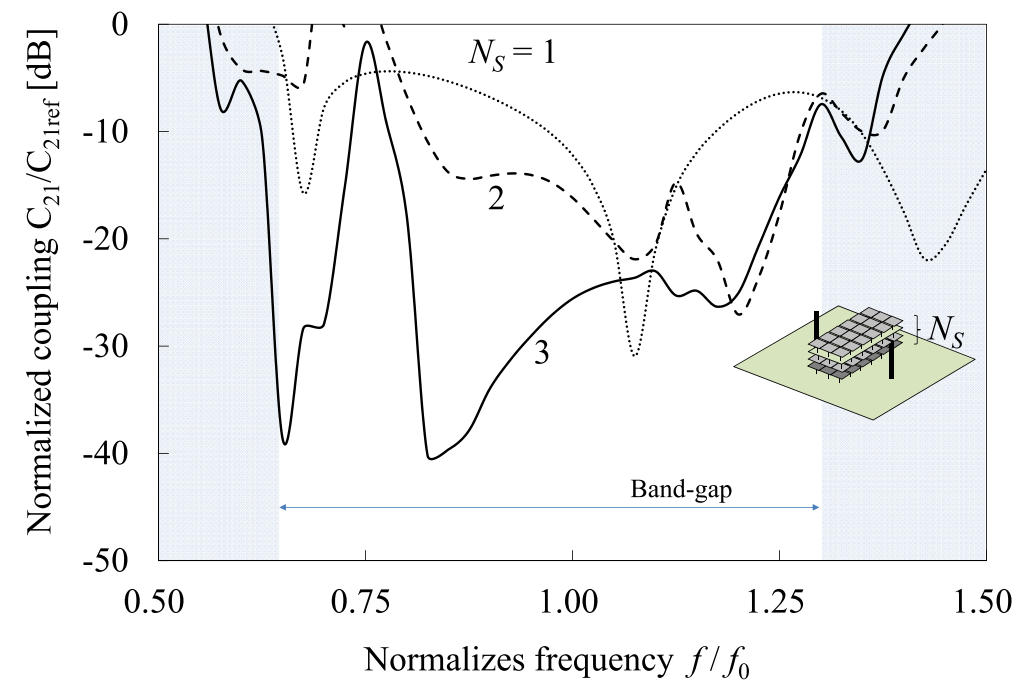

(a) Normalized coupling

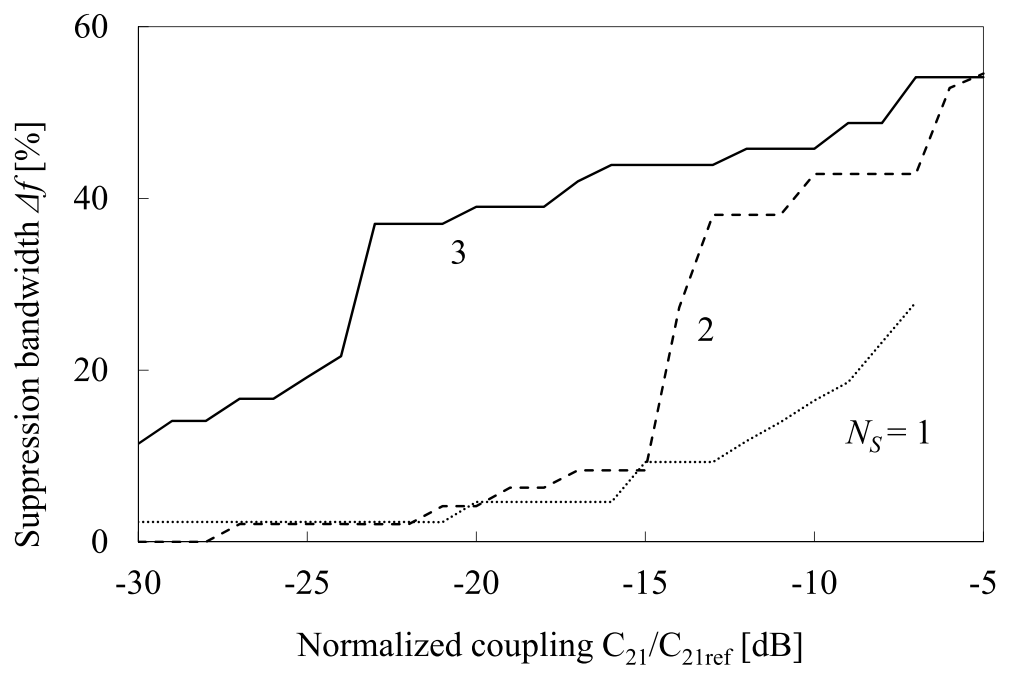

(b) Suppession bandwidth 
mutual coupling $C_{21} / C_{21 \text { ref }}$ and the coupling suppression bandwidth $\Delta f$. Here, the couplings $C_{21}$ and $C_{21 \text { ref }}$ are the coupling between antennas with the mushroom structure and without the structure. The coupling suppression bandwidth $\Delta f$ is defined as the frequency band where the normalized mutual coupling $C_{21} / C_{21 \text { ref }}$ become smaller than the specific value. The solid line and dashed line indicate the calculated results for the stacks $N_{S}=3$ and 2, respectively. For reference, the results for $N_{S}=1$, which is the case of conventional mushroom structure arranged model, is shown in dotted line.

It is found from Fig. 2(a) that the mutual coupling between the antennas is reduced by the mushroom structures regardless the number of the stacks $N_{S}$. The coupling suppression bandwidth is nearly equal to the bandwidth that the mushroom structure has band-gap characteristics. In case of $N_{S}=3$, we can obtain the coupling suppression effects of $40 \mathrm{~dB}$. As shown in Fig. 2(b), more wideband suppression effect can be achieved as the number of the stacks $N_{S}$ is increased. When the number of the stacks $N_{S}=3$ and the normalized coupling $C_{21} / C_{21 \text { ref }}=$ $-20 \mathrm{~dB}$, the suppression bandwidth $\Delta f=39 \%$, that is, the suppression effects of $20 \mathrm{~dB}$ or more can be obtained in the frequency band of $39 \%$. In summary, proposed mushroom structure can suppress the mutual coupling more than $2 \mathrm{D}$ arranged structure.

\section{Fabricated mushroom structure and experimental results}

In order to verify the above-mentioned calculated results, proposed mushroom structures was fabricated. Fig. 3(a), 3(b) and 3(c) show the photograph of monopole antennas, a conventional 2D arranged mushroom structure and the proposed multi-stacked mushroom structure. The size of the ground plane is $3.3 \lambda_{0} \times 3.3 \lambda_{0}$. The value of the other parameters is that utilizes in case of calculation. Fig. 3(d) shows the calculated and experimental coupling between monopole antennas. It is found that the coupling is suppressed by multi-stacked mushroom structure in measurement as well as in calculation. 


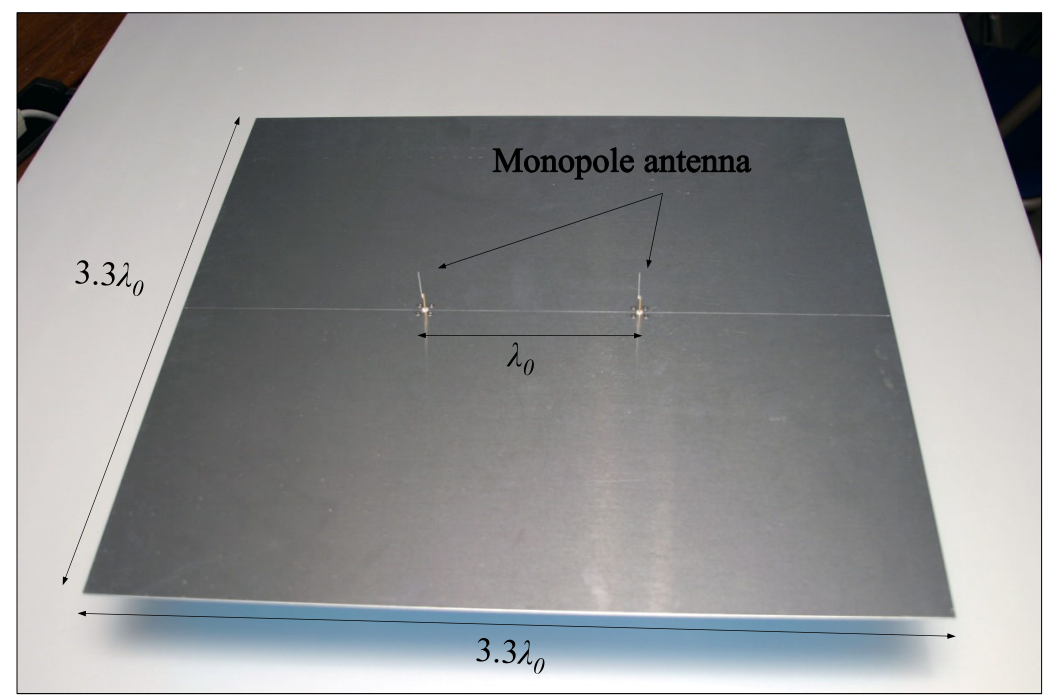

(a) Monopole antennas

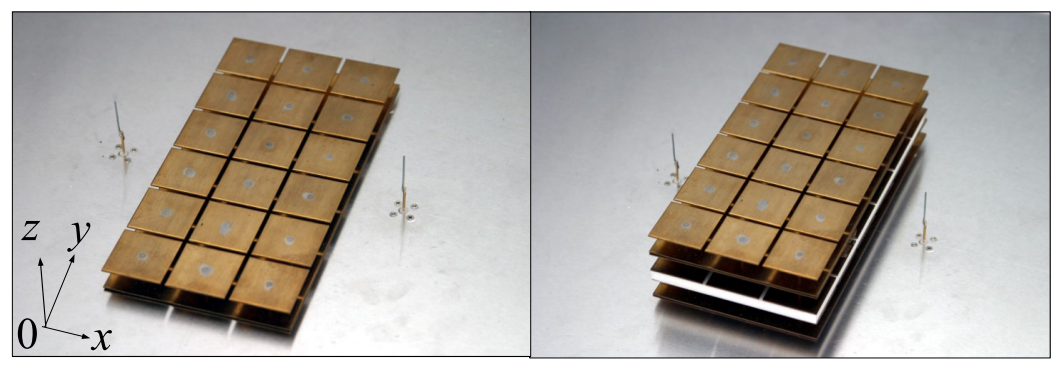

(b) Mushroom structure

(c) Multi-stacked mushroom structure

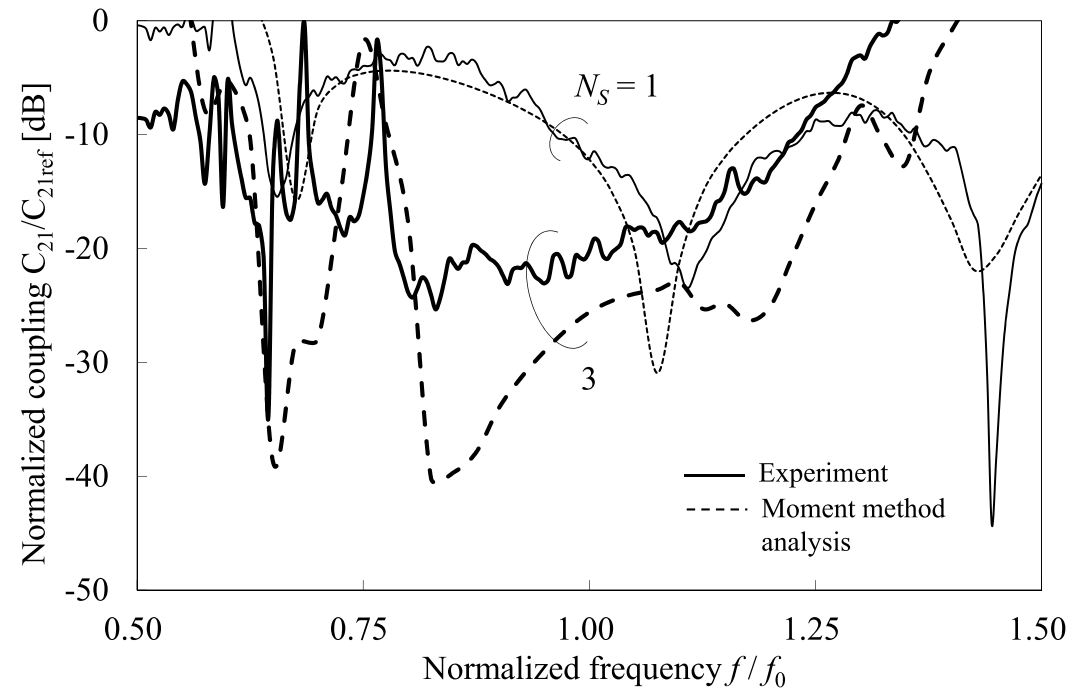

(d) Experimental results

Fig. 3. Suppression property of antenna coupling by fabricated mushroom structure.

\section{Conclusion}

Multi-stacked mushroom structures were proposed, and the coupling suppression property by the mushroom structures was discussed. Based on the calculated results, it was clarified that the proposed mushroom structure could suppress the mutual coupling more than conventional mushroom structures. In case of $d=\lambda_{0}$, the suppression effects of $20 \mathrm{~dB}$ or more could be achieved in the frequency band of $39 \%$. 